\title{
GROTHENDIECK-RIEMANN-ROCH AND THE MODULI OF ENRIQUES SURFACES
}

\author{
Georgios Pappas
}

\begin{abstract}
We give a short and "classical" proof of Borcherds' theorem that the moduli space of Enriques surfaces is quasi-affine. The use of the Borcherds' product is replaced in our proof by an application of the Grothendieck-Riemann-Roch theorem.
\end{abstract}

\section{1}

A (complex) Enriques surface is a projective smooth connected algebraic surface $Y$ over $\mathbf{C}$ with $\mathrm{H}^{1}\left(Y, \mathcal{O}_{Y}\right)=\mathrm{H}^{2}\left(Y, \mathcal{O}_{Y}\right)=(0),\left(\Omega_{Y}^{2}\right)^{\otimes 2} \simeq \mathcal{O}_{Y}$ but $\Omega_{Y}^{2} \not \mathcal{O}_{Y}([\mathrm{CD}])$. In this note we give a short proof of the fact that the coarse moduli space of complex Enriques surfaces is quasi-affine. This was first shown by Borcherds [B] using the denominator function of a generalized Kac-Moody superalgebra (the " $\Phi$-function"). From this, it follows (see [BKPS]) that any complete family of complex Enriques surfaces is isotrivial. Our observation here is that the ingredient of Borcherds' theory of infinite products can be completely removed from the proof and can be replaced by a simple use of the Grothendieck-Riemann-Roch theorem.

\section{2}

We refer the reader to $[\mathrm{CD}]$ and $[\mathrm{BPV}]$ for background on Enriques and $K 3$ surfaces. Let $L=E_{8}(-1) \oplus E_{8}(-1) \oplus H \oplus H \oplus H$ be the $K 3$ lattice with involution $\rho: L \rightarrow L$ given by

$$
\rho\left(\left(x, y, z_{1}, z_{2}, z_{3}\right)\right)=\left(y, x,-z_{1}, z_{3}, z_{2}\right) .
$$

Here $H=\mathbf{Z} \cdot e \oplus \mathbf{Z} \cdot f$ is the hyperbolic plane lattice with $\langle e, f\rangle=1$, and $E_{8}$ denotes the positive definite orthogonal lattice corresponding to the Dynkin diagram of type $E_{8}$. (The parentheses $(m)$ mean that we modify the pairing by multiplying it by the integer m.) Let $L^{+}=\{l \in L \mid \rho(l)=l\}, L^{-}=\{l \in L \mid \rho(l)=-l\}$. Then $L^{+}$is isometric to $E_{8}(-2) \oplus H(2)$; the unimodular lattice $L^{+}(1 / 2)$ can be identified with the Enriques lattice and is isometric to $E_{8}(-1) \oplus H$. The lattice $L^{-}$has signature $(2,10)$. We set

$$
\begin{gathered}
\Omega=\{\omega \in \mathbf{P}(L \otimes \mathbf{C}) \mid\langle\omega, \omega\rangle=0,\langle\omega, \bar{\omega}\rangle>0\}, \\
\Omega^{-}=\left\{\omega \in \mathbf{P}\left(L^{-} \otimes \mathbf{C}\right) \mid\langle\omega, \omega\rangle=0,\langle\omega, \bar{\omega}\rangle>0\right\} .
\end{gathered}
$$

for the 20-dimensional (resp. 10-dimensional) hermitian symmetric space for $O(3,19)$ (resp. for $O(2,10))$. ( $\Omega^{-}$has two 2 connected components.) Also let $O(L)^{\rho}=\{g \in$ $O(L) \mid g \cdot \rho=\rho \cdot g\}$,

$$
O(L)^{-}=\operatorname{restr}_{L^{-}}\left(O(L)^{\rho}\right)
$$

Received by the editors January 9, 2007.

*Partially supported by NSF Grant DMS05-01409. 
where by $O$ we denote the orthogonal group of automorphisms of the lattice that preserve the form. By work of Horikawa, $O(L)^{-}=O\left(L^{-}\right)$. For a vector $d$ of norm $\langle d, d\rangle=-2$ in $L^{-}$let $H_{d}$ be the divisor of points of $\Omega^{-}$represented by vectors orthogonal to $d$ and set $\Omega_{0}^{-}=\Omega^{-}-\left(\cup_{d} H_{d}\right)$. The group $O(L)^{-}=O\left(L^{-}\right)$acts on $\Omega_{0}^{-}$.

By the work of Namikawa and especially Horikawa $([\mathrm{H}],[\mathrm{BPV}]))$ on the Torelli theorem for Enriques surfaces, the (coarse) moduli variety $D^{0}$ of complex Enriques surfaces can be identified with the quotient

$$
D^{0}=\Omega_{0}^{-} / O\left(L^{-}\right) .
$$

(This has the structure of a quasi-projective variety over C.) There is no "universal" family of Enriques surfaces over $D^{0}$ but this can be remedied by passing to a suitable finite cover:

Proposition 2.1. There is a normal subgroup $\Gamma \subset O\left(L^{-}\right)$of finite index such that:

(i) The group $\Gamma$ acts freely on $\Omega_{0}^{-}$. The corresponding quotient

$$
D_{\Gamma}^{0}=\Omega_{0}^{-} / \Gamma
$$

is a smooth variety which supports a smooth family of Enriques surfaces $f: Y \rightarrow D_{\Gamma}^{0}$.

(ii) There is a family of K3-surfaces $g: X \rightarrow D_{\Gamma}^{0}$ with a free involution $\iota: X \rightarrow X$ which respects $g$ and is such that $Y=X /\langle\iota\rangle$. Denote by $p: X \rightarrow Y$ the covering morphism.

(iii) The line bundle of automorphic forms of weight 1 on $\Omega^{-}$(with fiber over the point $\omega \in \Omega^{-} \subset \mathbf{P}\left(L^{-} \otimes \mathbf{C}\right)$ given by the corresponding line in $\left.L^{-} \otimes \mathbf{C}\right)$ descends to the line bundle $\omega_{\Gamma}=g_{*}\left(\Omega_{X / D_{\Gamma}^{0}}^{2}\right)$ on $D_{\Gamma}^{0}$.

Proof. The existence of such a subgroup $\Gamma$ appears to be well-known to the experts; for the sake of completeness we sketch one possible construction here. Let us consider the vector $v=(0,0,0, e+f, e+f)$ in $L^{+} \subset L$; we have $\langle v, v\rangle=4$. Let us consider the groups $\Gamma_{v}=\{g \in O(L) \mid g(v)=v\}<O(L)$,

$$
\Gamma_{v}^{\rho}=\{g \in O(L) \mid \rho \cdot g=g \cdot \rho, g(v)=v\}<O(L), \quad \Gamma_{v}^{-}=\operatorname{restr}_{L^{-}}\left(\Gamma_{v}^{\rho}\right)<O\left(L^{-}\right) .
$$

(The structure of $\Gamma_{v}^{-}$is explained in [StI, Prop. 2.7].) For odd $n \geq 3$, we set $\Gamma_{n}=$ $\{g \in O(L) \mid g \equiv \operatorname{Id} \bmod n L\}$ and set $\Gamma_{v, n}:=\Gamma_{v} \cap \Gamma_{n}, \Gamma_{v, n}^{\rho}:=\Gamma_{v}^{\rho} \cap \Gamma_{n}$. Similarly, denote by $\Gamma_{v, n}^{-}$the restriction $\operatorname{restr}_{L^{-}}\left(\Gamma_{v, n}^{\rho}\right)$; using [StI, Prop. 2.7] and the fact that $n$ is odd we see that

$$
\Gamma_{v, n}^{-}=\Gamma_{v}^{-} \cap\left\{h \in O\left(L^{-}\right) \mid h \equiv \operatorname{Id} \bmod n\right\} .
$$

This is a normal subgroup of finite index in $\Gamma_{v}^{-}$. By a well-known lemma of Serre, $\Gamma_{n}$ and all our groups with a subscript $n$ have only trivial torsion. The group $\Gamma_{v, n}$ acts on the homogeneous space $\Omega_{v}=\left\{\omega \in \mathbf{P}\left((\mathbf{C} \cdot v)^{\perp}\right) \mid\langle\omega, \omega\rangle=0,\langle\omega, \bar{\omega}\rangle>0\right\}$ with trivial stabilizers. Using the period morphism and the Torelli theorem (e.g $[\mathrm{BR}],[\mathrm{Fr}])$, we can identify the smooth quasi-projective quotient $\Omega_{v} / \Gamma_{v, n}$ with the fine moduli space of polarized $K 3$ surfaces with a polarization of degree 4 and $n$-level structure. Now notice that the subgroup $\Gamma_{v, n}^{\rho}$ preserves $\Omega^{-} \subset \Omega_{v}$ and its action factors through $\Gamma_{v, n}^{\rho} \rightarrow \Gamma_{v, n}^{-}$. We have a morphism

$$
\Omega^{-} / \Gamma_{v, n}^{-} \rightarrow \Omega_{v} / \Gamma_{v, n} .
$$


The group $\Gamma_{v, n}^{-}$acts freely on $\Omega^{-}$. The quotient $\Omega^{-} / \Gamma_{v, n}^{-}$is also a smooth quasiprojective variety and supports a family $\tilde{g}: \tilde{X} \rightarrow \Omega^{-} / \Gamma_{v, n}^{-}$of $K 3$-surfaces (obtained by pulling back the universal family over $\Omega_{v} / \Gamma_{v, n}$ ). In addition, we see (using the Torelli theorem again) that the family supports an involution $\tilde{\iota}: \tilde{X} \rightarrow \tilde{X}$. Now restrict the family $\tilde{g}$ over the complement of the images of the divisors $H_{d}$, i.e over

$$
D_{\Gamma_{v, n}^{-}}^{0}=\left(\Omega^{-}-\left(\cup_{d} H_{d}\right)\right) / \Gamma_{v, n}^{-} .
$$

Using results of Nikulin we see as in $[\mathrm{Y}, \S 1]$ that the involution $\tilde{\iota}$ is fixed-point-free over this complement. Denote by $g: X \rightarrow D_{\Gamma_{v, n}^{-}}^{0}$ the corresponding (restricted) family of $K 3$ 's with involution $\iota: X \rightarrow X$. The family $f: X /\langle\iota\rangle \rightarrow D_{\Gamma_{v, n}^{-}}^{0}$ is a family of Enriques surfaces with the desired properties. For any subgroup $\Gamma \subset \Gamma_{v, n}^{-}$of finite index the families $f$ and $g$ pull back to families over $D_{\Gamma}^{0}$ with the same properties; this allows to make sure that $\Gamma$ can be taken to be normal in $O\left(L^{-}\right)$.

For simplicity of notation we will set $S=D^{0}, T=D_{\Gamma}^{0}$ and $\omega_{\Gamma}=\omega$.

The natural map $\pi: T \rightarrow S$ is a finite morphism of quasi-projective varieties and $S$ is identified with the quotient $T / G$ where $G:=O\left(L^{-}\right) / \Gamma$. Now consider the line bundle $\omega^{\otimes 2}$ over $T$.

Lemma 2.2. We have $f^{*}\left(\omega^{\otimes 2}\right) \simeq\left(\Omega_{Y / T}^{2}\right)^{\otimes 2}$ as line bundles on $Y$.

Proof. Since $f: Y \rightarrow T$ is a family of Enriques surfaces, the line bundle $\left(\Omega_{Y / T}^{2}\right)^{\otimes 2}$ is trivial along the fibers of $f$. Therefore, there is a line bundle $\delta$ on $T$ such that $\left(\Omega_{Y / T}^{2}\right)^{\otimes 2} \simeq f^{*}(\delta)$ and we have to show that $\delta \simeq \omega^{\otimes 2}$. Notice that

$$
g^{*} \delta \simeq p^{*} f^{*} \delta \simeq p^{*}\left(\left(\Omega_{Y / T}^{2}\right)^{\otimes 2}\right) \simeq\left(\Omega_{X / T}^{2}\right)^{\otimes 2} \simeq g^{*} \omega^{\otimes 2} .
$$

The result now follows by applying $\mathrm{R}^{0} g_{*}$ to both sides of $g^{*} \delta \simeq g^{*} \omega^{\otimes 2}$ after using the projection formula and $\mathrm{R}^{0} g_{*}\left(\mathcal{O}_{X}\right) \simeq \mathcal{O}_{S}$.

Now let us apply the Grothendieck-Riemann-Roch theorem ([Fu, Theorem 15.2]) to the proper morphism $f: Y \rightarrow T$ and to the trivial line bundle $\mathcal{O}_{Y}$ on $Y$. This gives

$$
\operatorname{ch}\left(\mathrm{R} f_{*} \mathcal{O}_{Y}\right)=f_{*}\left(\operatorname{ch}\left(\mathcal{O}_{Y}\right) \cdot \operatorname{Td}\left(T_{f}\right)\right)=f_{*}\left(\operatorname{Td}\left(T_{f}\right)\right)
$$

in the Chow ring with rational coefficients $\mathrm{CH}^{*}(T)_{\mathbf{Q}}:=\mathrm{CH}^{*}(T) \otimes_{\mathbf{Z}} \mathbf{Q}$. In this formula, ch is the Chern character and $\mathrm{Td}$ the Todd class. Also $\mathrm{R} f_{*} \mathcal{O}_{Y}$ denotes the (virtual) total cohomology bundle, and $T_{f}=\left(\Omega_{Y / T}^{1}\right)^{\vee}$ is the relative tangent bundle of the smooth morphism $f$. On the right hand side of the formula, $f_{*}: \mathrm{CH}^{*}(Y)_{\mathbf{Q}} \rightarrow$ $\mathrm{CH}^{*-2}(T)_{\mathbf{Q}}$ is the push forward homomorphism on Chow groups. Set $v_{1}$ for the class of $\omega$ in $\operatorname{Pic}(T)$. By the above lemma, we have $f^{*}\left(v_{1}\right)=-2 \cdot c_{1}\left(T_{f}\right)$. For simplicity, let us denote by $c_{i}=c_{i}\left(T_{f}\right)$ the Chern classes of the relative tangent bundle. By reading off the degree 1 component of the Grothendieck-Riemann-Roch identity (2.2) we find

$$
c_{1}\left(\mathrm{R} f_{*}\left(\mathcal{O}_{Y}\right)\right)=\frac{1}{24} \cdot f_{*}\left(c_{1} \cdot c_{2}\right)
$$

in $\operatorname{Pic}(T) \otimes \mathbf{Q}$. Since for the family of Enriques surfaces $f: Y \rightarrow T$, we have $\mathrm{R}^{0} f_{*}\left(\mathcal{O}_{Y}\right)=\mathcal{O}_{T}, \mathrm{R}^{i} f_{*}\left(\mathcal{O}_{Y}\right)=(0)$ if $i>0$, we have $\mathrm{R}_{*}\left(\mathcal{O}_{Y}\right)=\mathcal{O}_{T}$. Hence, the above gives

$$
f_{*}\left(c_{1} \cdot c_{2}\right)=0
$$


in $\operatorname{Pic}(T) \otimes_{\mathbf{z}} \mathbf{Q}$. Hence, we obtain

$$
0=2 \cdot f_{*}\left(c_{1} \cdot c_{2}\right)=-f_{*}\left(f_{*}\left(v_{1}\right) \cdot c_{2}\right)=-v_{1} \cdot f_{*}\left(c_{2}\right) .
$$

By Noether's fomula $f_{*}\left(c_{2}\right)=12$ in the Chow group of codimension 0 cycles $\mathrm{CH}^{0}(T)$ and so the class $v_{1}=c_{1}(\omega)$ is trivial in $\operatorname{Pic}(T) /$ torsion $\subset \operatorname{Pic}(T) \otimes_{\mathbf{Z}} \mathbf{Q}$. Therefore, the line bundle $\omega$ over $T$ is torsion.

Now notice that automorphic forms of weight $n$ on $D_{\Gamma}^{0}$ are by construction sections of

$$
\left(\pi_{*} \omega_{\Gamma}^{\otimes n}\right)^{G}
$$

over $S=D^{0}$ (i.e $G=O\left(L^{-}\right) / \Gamma$-invariant sections of $\omega^{\otimes n}=\omega_{\Gamma}^{\otimes n}$ over $T=D_{\Gamma}^{0}$ ). Since the line bundle $\omega$ is torsion and the group $G$ is finite, for $n$ sufficiently large and divisible, the line bundle $\omega_{\Gamma}^{\otimes n}$ has a nowhere vanishing $G$-invariant global section. On the other hand, by Baily-Borel ([BB]), for all sufficiently large and divisible $n$ the line bundle of automorphic forms of weight $n$ on $D^{0}$ is very ample. These two facts together imply that the moduli space $S=D^{0}$ is quasi-affine exactly as in [B]. As in loc. cit. it follows (using results of Sterk) that $D^{0}$ is in fact an affine variety with a copy of the affine line $\mathbf{C}$ removed.

Remark 2.3. The above argument applies to any family of (classical) Enriques surfaces $f: Y \rightarrow T$, even if $T$ is a variety over a field of positive characteristic. The conclusion is that $\mathrm{R}^{0} f_{*}\left(\left(\Omega_{Y / T}^{2}\right)^{\otimes 2}\right)$ is a torsion line bundle on $T$.

\section{Acknowledgment:}

The author is most grateful to I. Dolgachev for his comments.

\section{References}

[CD] F. Cossec and I. Dolgachev, Enriques surfaces. I. Progress in Mathematics, 76. Birkhäuser Boston, Inc., Boston, MA, 1989. x+397 pp.

[Fr] R. Friedman, A new proof of the global Torelli theorem for K3 surfaces. Ann. of Math. 120 (1984), no. 2, 237-269.

[Fu] W. Fulton, Intersection Theory. Ergebnisse der Mathematik und ihrer Grenzgebiete (3), 2. Springer-Verlag, Berlin, 1984. xi+470 pp.

[BB] W. L. Baily and A. Borel, Compactification of arithmetic quotients of bounded symmetric domains. Ann. of Math. 84 (1966), no. 2, 442-528.

[BPV] W. Barth, C. Peters, A. Van de Ven, Compact Complex Surfaces. Berlin: Springer 1984.

[B] R. Borcherds, The moduli space of Enriques surfaces and the fake Monster Lie superalgebra. Topology 35 (1996), no. 3, 699-710.

[BKPS] R. Borcherds, L. Katzarkov, T. Pantev, and N. I. Shepherd-Barron, Families of K3 surfaces. J. Algebraic Geom. 7 (1998), no. 1, 183-193.

[BR] D. Burns and M. Rapoport, On the Torelli problems for Kählerian K3 surfaces. Ann. Sci. Éc. Norm. Supér., IV. Sr. 8 (1975), 235-274.

[H] E. Horikawa, On the periods of Enriques surfaces, I, II. Math. Ann. 234 (1978) 73-108; 235 (1978), 217-246.

[Y] K. I. Yoshikawa, K3 surfaces with involution, equivariant analytic torsion, and automorphic forms on the moduli space. Invent. Math. 156 (2004), no. 1, 53-117.

[StI] H. Sterk, Compactifications of the period space of Enriques surfaces. I. Math. Z. 207 (1991), no. $1,1-36$.

Dept. of Mathematics, Michigan State University, E. Lansing, Mi 48824-1027, USA

E-mail address: pappas@math.msu.edu 